\title{
Editorial
}

\section{Social Media and Social Capital: Introduction to the Special Issue}

\author{
Sonja Utz * and Nicole Muscanell \\ Leibniz-Institut für Wissensmedien, Schleichstr. 6, 72076 Tübingen, Germany; \\ E-Mail: n.muscanell@iwm-tuebingen.de \\ * Author to whom correspondence should be addressed; E-Mail: s.utz@iwm-tuebingen.de; \\ Tel.: +49-7071-979-308; Fax: +49-7071-979-100.
}

Academic Editor: Gregor Wolbring

Received: 21 April 2015 / Accepted: 22 April 2015 / Published: 4 May 2015

Social media, especially social network sites (SNS) such as Facebook have grown rapidly in popularity in the last ten years. Facebook now has more than 1.3 billion users. Social media make it easier to maintain connections with strong ties, such as close friends and relatives, but also with weak ties, such as acquaintances or people only met once [1]. They also allow for new connections. Further, social media often blur audience boundaries (audiences are collapsed into one general audience) [2]. To make things even more complex, social media offer public and private communication features that can be further classified, for example, into directed communication (i.e., tagging, liking, or commenting) or more passive communication (i.e., silently consuming what is on your newsfeed) [3]. Unsurprisingly, scholars have thus wondered how social media might impact the nature of interpersonal relationships and ultimately the effects of social capital, i.e., the information and emotional support that people retrieve from their (online or offline) social network.

Traditional research on social capital has shown the benefits people can get from their social networks; strong ties provide them with emotional support (hence also bonding capital), and weak ties provide them with non-redundant information and different perspectives (bridging capital) [4,5]. However, these assumptions might no longer hold true on social media where different contexts and audiences collapse, individuals tend to have larger and more heterogeneous networks, and they can communicate in varied and newer ways than previously. This Special Issue brings together papers from different disciplines that focus on generating a current understanding about: (1) the benefits people seek and receive from their social media networks (i.e., informational, instrumental support, and/or emotional support); (2) how tie strength influences which benefits people receive; and (3) the 
underlying processes, e.g., the role of self-disclosure or relationship maintenance strategies. Further, this issue expands the literature on social media and social capital by including papers that examine more diverse samples, including participants from Germany, India, the UK, The Netherlands, and the U.S. It also extends the literature by examining other populations, in addition to student convenience samples (including immigrants and non-faculty university staff). Two papers also examine less studied SNS platforms, such as Orkut and Hyves.

As a starting point, the paper by Antheunis, Vanden Abeele, and Kanters [6] synthesizes prior empirical studies on Facebook use and social capital. They present evidence that the way in which individuals communicate via SNS matters. More specifically, directed communication and public broadcasting ( $v s$. passive consumption) strengthen bonding and bridging capital. Further, the available literature on the role of self-esteem in social capital provides evidence for the "poor get richer" theory [7], i.e., people with lower self-esteem seem to profit most from Facebook use. Importantly, Antheunis et al. [6] identify several theoretical, analytical and methodological issues that future research should consider, such as a clearer theoretical conceptualization of central constructs, a stronger consideration of the available features at the time of the study, the measurement of social capital, and the examination of more diverse samples.

Two papers focus on the perceived benefits people receive from Facebook and the role of tie strength $[8,9]$. Both papers examine social capital at the single contact level as opposed to the network level (i.e., by asking participants to respond about specific SNS friends). At a first glance the two studies seem to present contrasting results. Krämer et al. [8] do not find support for the classical assumption that weak ties provide non-redundant information, whereas strong ties provide emotional support. Instead, they find that strong ties are perceived to provide both types of support, more so than medium or weak ties do. The number of ties that provided emotional support also predicted overall bonding and bridging capital, and respondents were more willing to let the weaker contacts go. Vitak [9] tried to disentangle social, emotional and instrumental support, but found that the items all loaded onto one factor, indicating again that the classical assumptions from social capital theory do not hold anymore on social media. Similar to Krämer et al. [8], Vitak [9] finds that strong ties do provide more social support, but upon closer examination of more fine-grained communication strategies and affordances, she shows that weak ties profit more from directed communication and relationship maintenance strategies. Vitak [9] argues that the technological affordances (e.g., visibility, streamlined interactions) may augment the perceptions especially for weaker ties. She thus draws a different conclusion and focuses on ways how SNS can help people to get more benefits from their weak ties. Taken together, both papers show that individuals perceive that their strong ties are more likely to provide all forms of social support. However, sites like Facebook provide features that allow users to engage in more directed communication and to employ specific relationship management strategies which help them to get more social capital from their weak ties.

Two of the papers examine the role that SNS play for immigrants and migrants, thus focusing on less studied populations and SNS [10,11]. The examination of individuals who move either within or outside of their home country is an area that is novel and needed in the domain of social capital and social media. These individuals may particularly face issues that directly affect their ability to obtain or maintain social capital (i.e., maintaining contact with existing ties becomes more difficult and they may have to bridge cultural boundaries in order to form new relationships). Again, similar to the previous 
studies, tie strength plays a central role, but now as a dependent variable. Binder and Sutcliffe [10] examined the effects of alternating the use of two SNS. They examined Indian nationals either migrating within India (study 1) or to a different country (study 2) and compared these with Indian people who did not move to a different place or country. Orkut is the dominant SNS in India, but many respondents additionally used Facebook. Differences in network size between migrants and non-migrants were explained by alternating SNS use, such that these differences diminish for those who use two SNS. Thus, this paper shows that alternating SNS use is a compensatory strategy that can help migrants to maintain and extend their network. Damian and van Ingen [11] similarly examined immigrants, but with a different population and different platforms. They focused on the immigrants in the Netherlands who used Facebook and/or Hyves (a Dutch SNS popular at the time of the study). They found that SNS users had more outgroup ties (i.e., ties with people from a different nationality than their own) among their five strongest ties and frequency of SNS use positively predicted satisfaction with relationships. Surprisingly though, this effect was independent from emotional self-disclosure. Taken together, these two studies show that immigrants who use SNS do not have more strong ties, but specifically more heterogeneous strong ties (i.e., more outgroup members). This could be seen as an indicator for better integration into the new home, especially when one considers that it is still the strong ties that provide more support.

Finally, Moll, Pieschl, and Bromme [12] take a look at the underlying processes explaining self-disclosure on online social networks. Similar to Damian and van Ingen [11], Moll et al. [12] argue that self-disclosure is an important process for building and maintaining ties, especially because it allows for a certain level of trust to be reached, which facilitates the exchange of social capital. Damian and van Ingen [11] did not find evidence for the role of self-disclosure, but they used panel data and therefore had a very general trait level measurement of self-disclosure. Moll et al. [12] take into account the (semi-)public nature of communication on social media and privacy issues. They present a theoretical model explaining cognitively how people regulate privacy online and how this might influence self-disclosure. In their subjective collective privacy theory (cp theory), they claim that SNS users are in a state of default trust and that they infer risk from (invalid) cues, which further reinforces the trust mode. They assume that SNS users often have experienced helplessness (not being able to control their data), information overload, and diffuse audience reactions and that this leads to the experience of collective privacy - the belief that others will not read everything and not bother about everything they have posted. In this default trust mode, people have problems remembering what they have posted to whom and with which privacy settings. Moll et al. [12] provide some first evidence for this theory and overall they suggest that vigilant users should be able to more adequately regulate their privacy boundaries and thus self-disclose based on their direct needs. This type of usage could thus enhance social capital.

Overall, this Special Issue provides a current look at several aspects of social capital as it pertains to social network sites. Together, these papers demonstrate that the boundaries between emotional and informational support may be blurred when it comes to SNS use. Strong ties are perceived as providing more emotional and instrumental support. However, the affordances of SNS allow users to engage in specific relationship maintenance strategies that can also help them to retrieve benefits from weaker ties. SNS use might also be especially useful for immigrants or migrants (those who relocate afar to new homes) and in particular these sites might leverage the playing field when it comes to social 
capital by allowing individuals both to maintain and extend their networks (i.e., with outgroup members). Finally, from these papers, several issues and areas of future research on social media and social capital are apparent (in addition to those addressed by Antheunis et al. [6]). First, future research should keep in mind the affordances that different SNS offer and that the way in which individuals perceive and utilize SNS are important moderators/mediators that predict one's ability to gain or maintain social capital. Second, the role of self-disclosure is still somewhat unclear and should be examined further, in addition to identifying other potential mediating variables that explain why and how people obtain more or less social capital from their SNS usage/networks. Third, these studies illustrate the importance of examining social capital and social media utilizing multiple methods (e.g., especially by using more fine-grained measures such as single contact level measures, measures of perceptions, behavioral measures, and by identifying specific types of communication and behaviors). Future research can further build upon the literature by examining both new and established measures and identifying which types of measures are best suited for assessing social capital derived from social media. To conclude, we see this area of research as very promising; especially given that each of the papers in this issue suggest that social media have potential to help people to get more out of their social networks. There are plenty of directions for research and it will be important to continue further exploring the potential benefits that can be derived from social media and other communication technology, especially as technology continues to change rapidly.

\section{References}

1. Ellison, N.B.; Steinfield, C.; Lampe, C. The benefits of Facebook "friends:" Social capital and college students' use of online social network sites. J. Comput. Commun. 2007, 12, 1143-1168.

2. Marwick, A.E.; boyd, d. I tweet honestly, I tweet passionately: Twitter users, context collapse, and the imagined audience. New Media Soc. 2011, 13, 114-133.

3. Burke, M.; Kraut, R.; Marlow, C. Social capital on facebook: Differentiating uses and users. In Proceedings of the SIGCHI Conference on Human Factors in Computing Systems; ACM: New York, NY, USA, 2011; pp. 571-580.

4. Granovetter, M.S. The strength of weak ties. Am. J. Sociol. 1973, 78, 1360-1380.

5. Putnam, R.D. Bowling Alone: The Collapse and Revival of American Community; Simon and Schuster: New York, NY, USA, 2000.

6. Antheunis, M.L.; Vanden Abeele, M.M.P.; Kanters, S. The Impact of Facebook Use on Micro-Level Social Capital: A Synthesis. Societies 2015, 5, 399-419.

7. Zywica, J.; Danowski, J. The Faces of Facebookers: Investigating social enhancement and social compensation hypotheses; predicting facebook and offline popularity from sociability and self-esteem, and mapping the meanings of popularity with semantic networks. J. Comput. Commun. 2008, 14, 1-34.

8. Krämer, N.C.; Rösner, L.; Eimler, S.; Winter, S.; Neubaum, G. Let the Weakest Link Go! Empirical Explorations on the Relative Importance of Weak and Strong Ties on Social Networking Sites. Societies 2014, 4, 785-809.

9. Vitak, J. Unpacking Social Media's Role in Resource Provision: Variations across Relational and Communicative Properties. Societies 2014, 4, 561-586. 
10. Binder, J.; Sutcliffe, A.G. The Best of Both Worlds? Online Ties and the Alternating Use of Social Network Sites in the Context of Migration. Societies 2014, 4, 753-769.

11. Damian, E.; van Ingen, E. Social Network Sites Usage and Personal Relations of Migrants. Societies 2014, 4, 640-653.

12. Moll, R.; Pieschl, S.; Bromme, R. Trust into Collective Privacy? The Role of Subjective Theories for Self-Disclosure in Online Communication. Societies 2014, 4, 770-784.

(C) 2015 by the authors; licensee MDPI, Basel, Switzerland. This article is an open access article distributed under the terms and conditions of the Creative Commons Attribution license (http://creativecommons.org/licenses/by/4.0/). 